\title{
Slenderize Your Body: Application and Evaluation of Anatomy in Apparel Design
}

\author{
Yan Cui ${ }^{1,2}$, Xiaogang Liu ${ }^{1} *$ \\ ${ }^{1}$ College of Fashion and Design, Donghua University, Shanghai 200051, China \\ ${ }^{2}$ Cornell University, Fiber Science and Apparel Design, Ithaca, NY, USA
}

\begin{abstract}
The idea of thinness as beauty is still the dominant ideology of modern women, and body modification through clothing is one of the main motivations for women's clothing consumption. Denim, as a primary category of body shaping apparel, has been gaining attention from brands and developers in recent years. This paper investigates an ergonomics-based approach for slim-fit denim garments that combines aesthetic plastic and stereovision into the fashion design. Ninety-seven female subjects with a background in fashion design were studied through data visualization and statistical analysis. Women's perception of a denim clothing line with anatomy design factors (ADF) was studied. The results reveal that ADF has a positive effect on the visual perception of female consumers and can help subjects to visually optimize their body shape, increase their body satisfaction and purchase intent. This study provides theoretical and data support for the integration of fashion art and sustainable innovation.
\end{abstract}

\section{Introduction}

Women's quest for a slimmer figure continues to be a global trend. Nowadays, the average female weight has tended to be rising. However, the mainstream cultural expectations for ideal feminine beauty was contrary to the increasing trend of average female weight, and this confliction makes women keep struggling between their perceptions of actual-self and internal ideal-self [1]. A compelling example was that young females in Hong Kong pay more attention to their diets even though they have had slender body shape [2]. In the prior study about the female body image of Korea and Hong Kong, the perception of "ideal beauty" across different cultures has been entirely consistent at the present days has some cross-cultural consistency to a certain extent [3]. The ideal female image forms a cross-cultural standard due to the uninterrupted impact of Asian culture on western countries [4].

Female consumers try to pursuing a perfect body in various ways, and the same is true for clothing consumption [5]. Throughout history, clothing consumption has not only fulfilled a person's functional needs, such as keeping warm and covering the body. Another form of the functionality of clothing products is called soft skill [6]; it includes the emotional and aesthetic needs of consumers. The primary function of denim as a typical garment was to flatter the figure. The "China Jeans Market Research and Development Prospects (2018-2025)" report shows that $86.3 \%$ wear jeans regularly, and only $1.3 \%$ do not wear jeans. Denim products have become a fashion sign for young people. The data reveals that only $13.5 \%$ of people prefer to wear casual jeans, while $64.3 \%$ prefer skinny jeans. With the rise in aesthetic requirements, female consumers are becoming higher demanding of their body-shape. As a result, more and more jeans brands were taking fit and optimizing body shape as one of their essential design criteria. For example, Levi's "Lady Style" collection has a smaller back patch pocket with a heart-shaped back piece, which was higher than the rest of the brand's jeans and was cut in a princess cut to create a buttock lift. However, these design methods remain in the realm of traditional clothing design, where designers shape the body by changing the shape of cut pieces, raising the waistline, or even using highly stretchable fabrics. The denim garment used in this research explored a new design methodology, which combined a series of anatomical shadows of ideal body image with washing area and optical illusion patterns to optimize the wearer's body shape on visual perception [7]. Anatomy design method incorporates visual principles to alter the cut of the denim garment; all of these design elements help the wearer to optimize their figure. This paper examines the ability of new design methods to optimize stature and its impact on consumer preferences through statistical analysis and data visualization analysis.

\section{Methodology}

In order to understand Asian female's perceptions of denim wears in China, especially for Urban Households, multiple statistical analysis methods were used in this research. A professional online questionnaire collection

*Correspondence: $\operatorname{liuxg@\text {dhu.edu.cn}}$ 
website issued the questionnaires. Participants can fill in the questionnaire either by the webpage or Mobile client. A total of 20 pre-tests were conducted to assess the efficacy and applicability of the questionnaire for denim wears. Minor revisions were made based on the observations and results of the pre-test. The final version of the questionnaire was designed and organized into eight sections [8, 9]: basic information, subjects' subjective perceptions of the new design; design evaluation of three types of clothing; the relationship between subjects' viewing perspectives and weight loss effects; the relationship between weight loss effects and willingness to purchase. Five-point Likert scale questions (anchored from strongly disagree $=1,2,3,4,5$ to strongly agree) were used to this survey combining with the single choice question, multiple-choice questions, and open-ended questions. The selection of samples follows diversity and representative: the samples should contain primary types of denim wears; they should be represented the typical garments in the denim market. Thus, a denim garment collection (5 dress, 2 short jackets, and 15 jeans.) was selected from the current market in this study, which applied to the anatomy design method. The test garments use four major design factors $[7,10]$ : anatomy shading, anatomy patterning, and anatomy warping (Figure 1). The principle of the Anatomy design method shown in figure 1 .
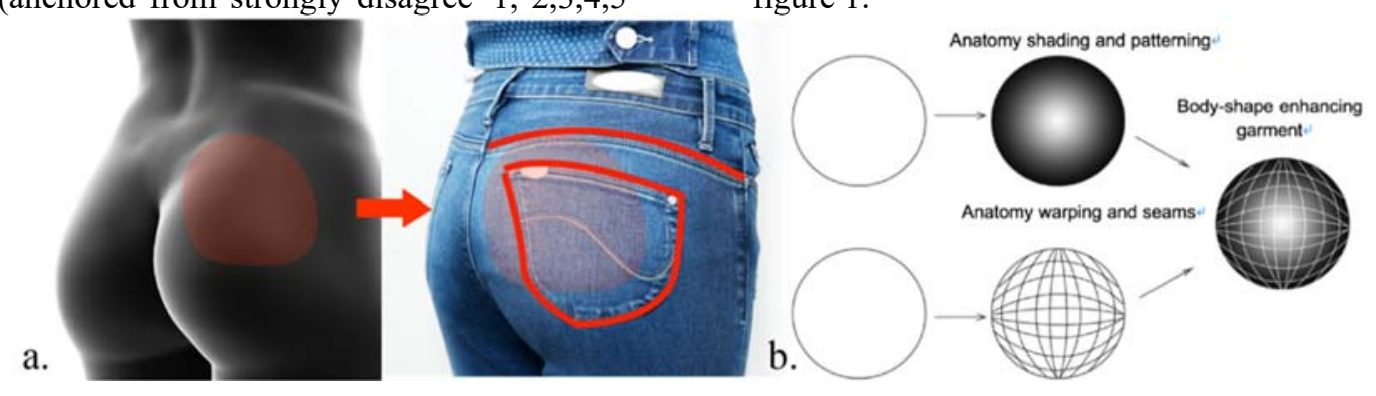

Figure 1. (a) left was a medically accurate illustration of female butt, the highlights area on this standard image were extracted (red area) and then applied it on jeans' design as washing patterns. In this way, the designer mapped the ideal body image onto the human body, creating a slim effect of garments. The effect helps the wearer improve body satisfaction. The red line corresponds to anatomy warping. (b) The design principle of the anatomy design method was to apply anatomy shading \& patterning and anatomy warping \& seams on the garment at the same time, which superimposed to form a strong three-dimensional visual perception [7, 11].

All participants were females and were recruited from a University in the Yangtze River delta area either at campuses or via personal contact by the researcher and research assistants, 96.91\% of them came from Shanghai, based on the geographic analysis. Test garments were carried out by 100 female participants for the sake of result representativeness and authoritative, $96.9 \%$ of them in the age range of 20 to 30 years old with a standard deviation of $0.56(n=94)$. All of them were selected from design-related majors with relatively higher aesthetic sense and professional knowledge background. $97.9 \%$ of them were undergraduate students or above with a standard deviation of 0.57 . During testing, 104 questionnaires were issued in total. Seven of them were excluded from the dataset due to inadequate responses or repeated submit, leaving a total of 97 valid questionnaires (93.27\% return rate).

\section{Results and Discussion}

\subsection{Subjective perceptions of the anatomy design method}

An open-ended question was set in the test: please state the main reason why you choose this product. Then, with the online visualization tool Voyant Tools, the open-ended questions were analyzed to find out the testers' subjective feelings about the test garments. The corpus has 362 total words and 162 unique word forms, and vocabulary Density was 0.448 . Most frequent words in the corpus were thin (11), good (10), jeans (9), looking (9), and comfortable (7). They were combining the results of cirrus, trends, and the original corpus (Figure 2a, b). The study found that subjects' subjective feelings about the test clothing were "thin, good-looking, and comfortable, " following with "longer leg" and "slim upper bod." Links' results reveal the relationships and distances between words. When "thin" was chosen as the core word, the words associated with it were "legs, looks, longer, beautiful, and design" In combination with the original corpus, it was found that when a garment flatters the wearer's figure, they perceive it as elegantly designed (Figure 2c). Furthermore, Bubblelines results indicate that the words thin and good-looking were highly overlapping. Garments' slim effect was directly related to consumers' subjective feelings of good looking, which means that "thinness as beauty" was still the mainstream thinking of young women. 


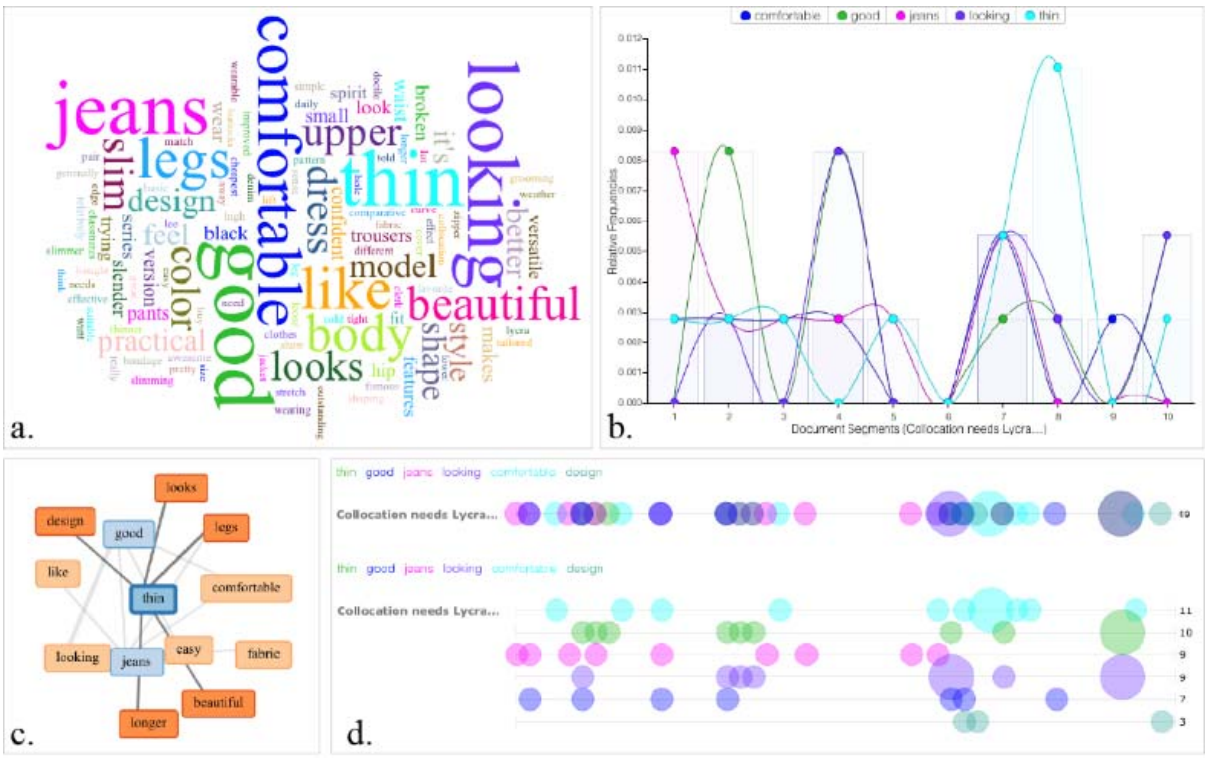

Figure 2. Data visualization and analysis results (Voyant Tools). (a) Cirrus analysis, (b) Trends analysis, (c) Links analysis, (d) Bubblelines analysis.

\subsection{The primary site of action of body- enhancing effect for three types of garments}

A multiple-choice analysis of where the three product categories specifically optimized body shape led to the following conclusions: jeans optimized the subjects' hips and Legs, visually curvy the hips, and slim the legs. The dress optimizes the subject's waist and abdomen, visually tapers the waist and conceals abdominal fat. The jacket optimized the subject's arms and abdomen, visually tapers the arms and also hides the belly fat.
Overall, the jeans could optimize the whole body evenly, basically at $45 \%$ or more. On the other hand, Dresses and jackets can optimize mainly on individual parts. Particularly, $77.78 \%$ of the participants believe that the jacket modified their waist curve, while for the rest part was apparent. Generally, jeans were better than the remaining two types of clothing in terms of overall optimization.

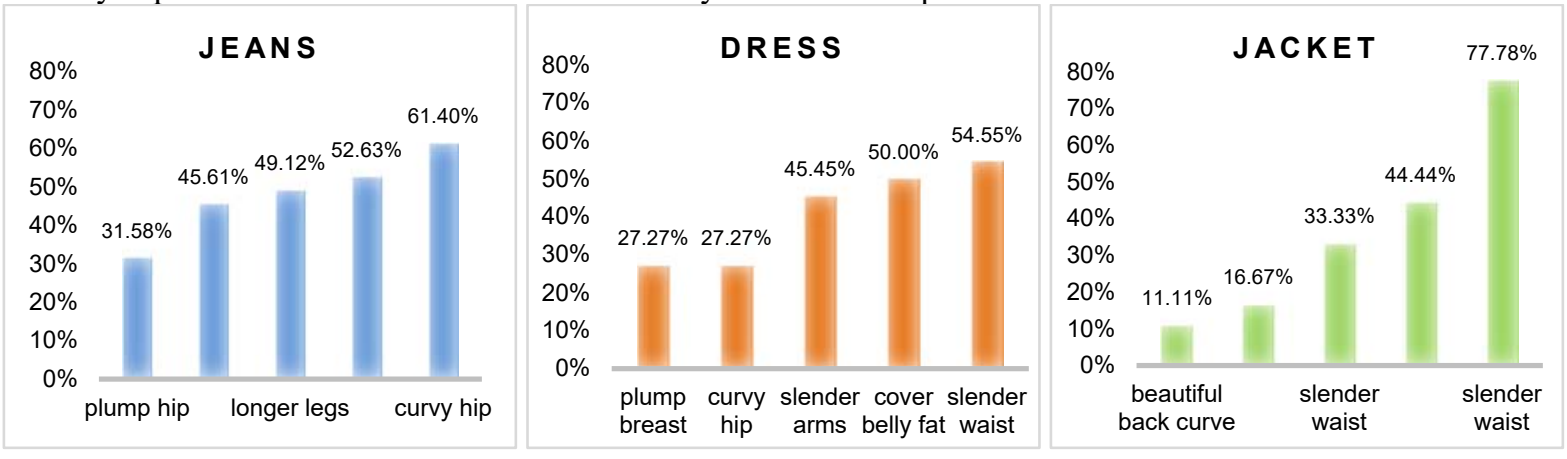

Figure 3. The primary site of action of body-enhancing effect for three types of garments.

\subsection{Consumer's viewing-angle verse anatomy denim garments' body-shape enhancing ability}

The test garments were designed with a series of anatomy design factors (ADF). All of them were twodimensional patterns or seams before the garments were tried on human bodies. The original flat design factors would turn to three-dimensional elements after putting on the body. It was unclear whether the stereoscopic illusion function of ADF would increase or not due to garments' display state. Therefore, the main research purpose of this part was to explore whether the display mode of garments would affect the efficacy of ADF, and further to find out the best display mode for tested garments. It was known that Asian female consumers prefer a slimmer body-shape; we presumed that the weight-reduction effect of test garment was highly correlated with the body-shape enhancing ability. In this section, the visually weight-reduction effect was regarded as the body-shape enhancing ability. The result of this section showed that the weight reduction effect from mirror viewing angle was better than selfobservation. $65.98 \%$ of females believe the test 
garments have $1 \mathrm{~kg}$ to $3 \mathrm{~kg}$ weight-loss effect on visual perception by self-observation and $67.10 \%$ by mirror observation. In addition, the $3 \mathrm{~kg}$ to $5 \mathrm{~kg}$ weight-loss effect increased from $4.12 \%$ (self-observation) to effect from the mirror was greater than self-observation $(\mathrm{t}=5.57, \mathrm{P}<0.01) \quad($ table 1$)$
$17.53 \%$ (mirror). And 0kg weight-loss effect reduced from $29.9 \%$ (self-observation) to $14.43 \%$ (mirror). The paired samples T-test results shown the weight-loss

Table 1. The paired samples T-test of weight-loss effect for different viewing angle.

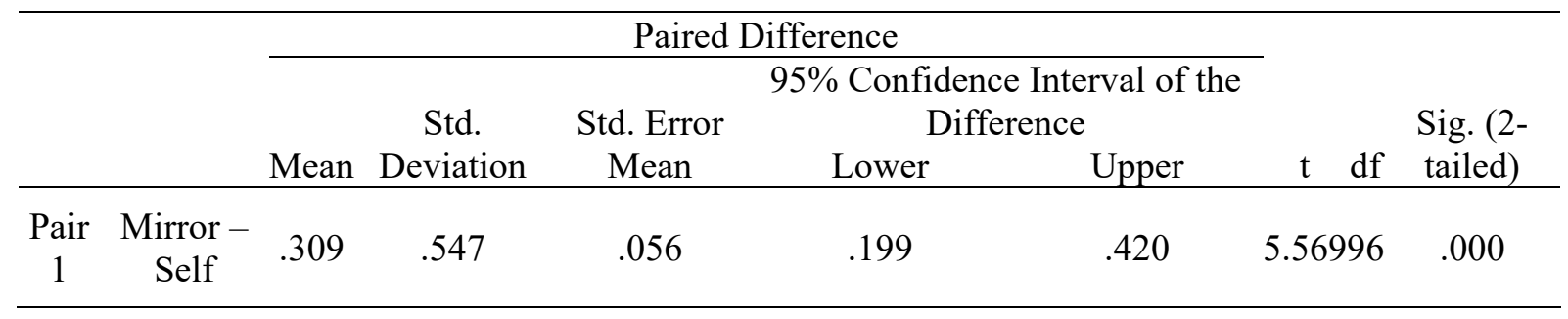

Besides, this section also tested the visual perception of other observers for tested garments, which would affect the consumer satisfaction assessment of garments. The comments or compliments from others would influence the self-satisfaction of social individuals. With the help of others opinion, subjects feel their actual-Self State were closer to ought-self state, and this would pass on this satisfaction to the clothing they wear, thereby consumers' satisfaction towards testing garments were increased. The result revealed that $73.2 \%$ of the subjects were told that they looked thinner than usual. The data were found to be unaligned by the tests of variance congruence of OTHERS(From others observation, how many people tell you that you have a better body size and shape?) and WTB (want to buy), so using SPSS Kruskal-Wallis H-test analysis of the relationship between OTHERS and WTB. Based on the hypothesis

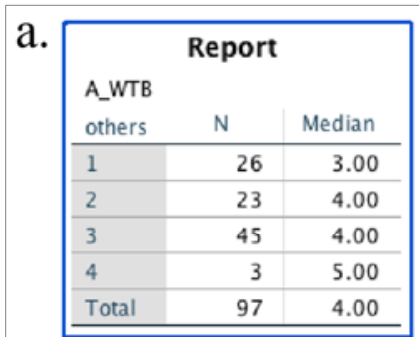

$1=$ zero people told subjects she looks thinne $2=1$ people told subjects she looks thinner $3=2-3$ people told subjects she looks thinner $4=4$ or more people told subjects she looks thinner d. Pairwise Comparisons of others

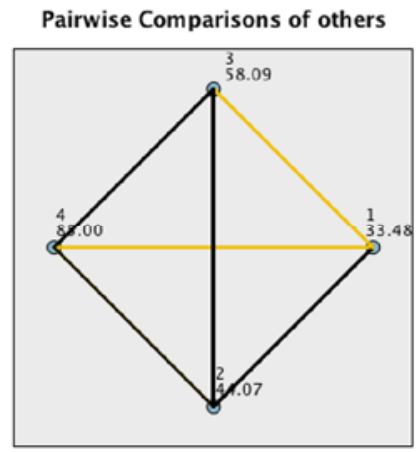

test summary, it can be assumed that the distribution of WTB was not identical across groups. The difference was statistically significant $(\mathrm{H}=20.691, \mathrm{P}=0.002)$; The median of WTB was 3 in the 0 person group $(n=26)$; The median of WTB was 4 in 1 person group $(n=22)$ and 2-3 person group $(n=45)$; and the median of WTB was 5 in $4+$ person group $(n=3)$. Significance values have been adjusted by the Bonferroni correction for multiple tests. The distribution of WTB scores was statistically significant between the 0 and 2-3 person groups (Adj. $\mathrm{P}=0.001$ ), 0 and $4+$ person groups (Adj. $\mathrm{P}=0.008$ ), with no difference between the other groups. This means that when less than one person tells the tester looks thinner, it does not affect the consumer' s desire to buy, but more than two people stating the same information will change the consumer's purchasing willingness.

\begin{tabular}{|c|c|c|c|c|c|}
\hline $\begin{array}{l}\text { Sample 1 } \\
\text {-Samp... }\end{array}$ & $\begin{array}{c}\text { Test } \\
\text { Statistic }\end{array}$ & $\underset{\text { Error }}{\text { Std. }} \vec{\nabla}$ & $\begin{array}{l}\text { Std. Test } \\
\text { Statistic }\end{array}$ & Sig. $\nexists$ & Adj.Sig. \\
\hline $1-2$ & -10.584 & 7.559 & -1.400 & .161 & .969 \\
\hline $1-3$ & -24.608 & 6.505 & -3.783 & .000 & .001 \\
\hline $1-4$ & -51.519 & 16.101 & -3.200 & .001 & .008 \\
\hline $2-3$ & -14.024 & 6.768 & -2.072 & .038 & .230 \\
\hline $2-4$ & -40.935 & 16.209 & -2.525 & .012 & .069 \\
\hline $3-4$ & -26.911 & 15.746 & -1.709 & .087 & .525 \\
\hline \multicolumn{6}{|c|}{$\begin{array}{l}\text { Each row tests the null hypothesis that the Sample } 1 \text { and } \\
\text { Sample } 2 \text { distributions are the same. } \\
\text { Asymptotic significances ( } 2 \text {-sided tests) are displayed. The } \\
\text { significance level is .05. } \\
\text { Significance values have been adjusted by the Bonferroni } \\
\text { correction for multiple tests. }\end{array}$} \\
\hline
\end{tabular}

Figure 4. (a) Median report between OTHERS and WTB, (b) \& (c) pairwise comparisons of OTHERS.

The above results exposed anatomy design method was universal for typical clothing styles. This section further confirms the importance that young female consumers place on the slimming effect of clothing. At the same time, the anatomy design method visually gives the wearer a weight-loss effect. This effect has been verified not only by the wearer themselves but also by others who have observed wearers. The results show that the anatomy design method has a uniform and stable body modification effect on both others' perceptions and self-perceptions. Furthermore, tested garments had a better weight-reducing effect after trying on, which means the ADF played a better role in three-dimensional situations. In consequence, the best way to display tested garment was stereo exhibiting rather than hanging them up. Meanwhile, trying to ask consumers to wear such garments can promote their satisfaction. In order to identify the relationship between garment shaping ability and consumers' willingness to purchase, this section calculates the correlation between weight reduction effect and willingness to purchase using correlation analysis.

The correlation analysis indicated a direct correlation between body-shape enhancing ability and consumer behavior and preference (stage 2). All of the correlation coefficients were significant than zero, which means the body-shape enhancing ability of testing garments have a positive influence on female 
consumers' behavior and preference $(p<0.01)$. Specifically, female consumers' preference was directly associated with their mirror image improvement (Correlation coefficient $=0.503, \mathrm{p}<0.01$ ). Among three viewing angles, mirror image has the most significant impact on consumer preference, following with friends' compliments and self-observation.

Table 2. Correlation analysis depicting body-shape enhancing ability influence on female consumer behavior and preference

\begin{tabular}{cccc}
\hline & Self & Mirror & Others \\
\hline Visual difference & $0.417^{* *}$ & $0.487^{* *}$ & $0.451^{* *}$ \\
Ideal body shape & $0.411^{* *}$ & $0.495^{* *}$ & $0.482^{* *}$ \\
WTB & $0.416^{* *}$ & $0.503^{* *}$ & $0.447^{* *}$ \\
How much & $0.225^{* *}$ & $0.266^{* *}$ & $0.313^{* *}$ \\
\hline
\end{tabular}

$* \mathrm{p}<0.05 * * \mathrm{p}<0.01$

\section{Conclusion}

This paper investigates a design method developed for slim denim clothing that incorporates human ergonomics, aesthetic plastic, and visual perception theory. Ninety-seven female subjects with fashion design or related background were invited to evaluate the products. A complete clothing collection was selected as test garment samples to study women's subjective perceptions of the anatomy design method through data visualization and statistical analysis. The relationship between the observation perspective and the garment shaping ability was calculated. Finally, the relationship between garment shaping ability and consumer purchase intention was analyzed. The study found that the participants' attitudes toward the anatomy design method were as follows Positive. They felt that tested garments left them with subjective feelings of thin, good-looking, and comfortable. The subject's perception depending on the observation angle and is better observed through a mirror than self-observation. Also, praise from others promotes consumers' willingness to purchase when more than two people have positive comments about the consumer. The willingness to buy changed from neutral to wanting to buy. At the same time, consumers perceived the test garments as visually different from other products, whether in a mirror view, self-observation, or evaluation by others, and It can help it create the perfect body. Moreover, the willingness to purchase most increases with the ability of the garment to shape the body. This study provides case studies and data to support the interdisciplinary application of apparel design for the development of slim-fit denim, and it also contributes to the fusion of fashion art and sustainable innovation.

\section{Acknowledgment}

The authors acknowledge the financial support of this work from the Discipline construction project (improving independent innovation and social service capabilities) under award number 107-08-0241028 from Shanghai Style Fashion Design \&Value Creation Collaborative Innovation Center.

\section{References}

1. Saraceni R, Russell-Mayhew S. Cultural expectations of thinness in women: A partial replication and update of magazine content. Eating and Weight Disorders-Studies on Anorexia, Bulimia and Obesity 2007;12(3):e68-e74.

2. Lee S, Lee AM. Disordered eating in three communities of China: A comparative study of female high school students in Hong Kong, Shenzhen, and rural Hunan. International Journal of Eating Disorders. 2000;27(3):317-27.

3. Hsu H-J, Burns LD. Clothing evaluative criteria: A cross-national comparison of Taiwanese and United States consumers. Clothing and Textiles Research Journal. 2002;20(4):246-52.

4. DeLong M, LaBat K, Nelson N, Koh A, Kim Y. Global products, global markets: Jeans in Korea and the United States. Clothing and Textiles Research Journal. 2002;20(4):238-45.

5. Cui Y, Dai Y, Liu X, editors. The Influence of Evaluation Subject Visual Perception and Fitting Experience on Consumer Preference for the BodyEnhancing Garment. International Textile and Apparel Association Annual Conference Proceedings; 2019: Iowa State University Digital Press.

6. McDonagh-Philp D, Lebbon C. The emotional domain in product design. The Design Journal. 2000;3(1):31-43.

7. Peshek DJ, Mark JT, Marion B, Stephens K, Hoffman D, Zades SH, inventors; Google Patents, assignee. Body-enhancing garment and garment design2019.

8. Patterson PG, Spreng RA. Modelling the relationship between perceived value, satisfaction and repurchase intentions in a business-to-business, services context: an empirical examination. International Journal of service Industry management. 1997.

9. Alexander M, Jo Connell L, Beth Presley A. Clothing fit preferences of young female adult consumers. International Journal of Clothing Science and Technology. 2005;17(1):52-64.

10. Hoffman D, Peshek DJ, Dull SF, Zades SH, Fisher RON, Mark JT, et al., inventors; Google Patents, assignee. Anatomy shading for garments2019.

11. Cuenca-Guerra R, Quezada J. What makes buttocks beautiful? A review and classification of the 
determinants of gluteal beauty and the surgical techniques to achieve them. Aesthetic Plast Surg. 2004;28(5):340-7. 